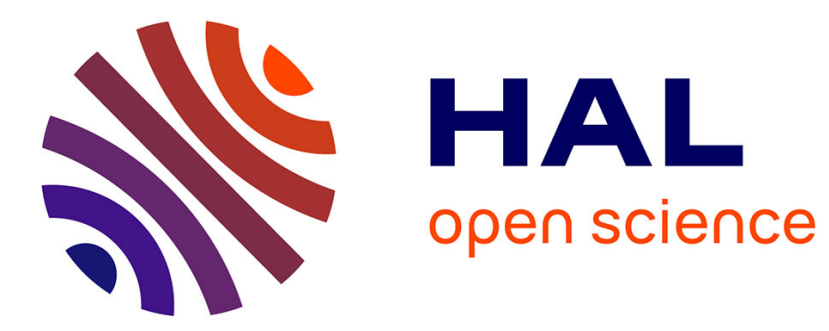

\title{
Stéphane Beaud et Gérard Mauger (dir.), Une génération sacrifiée ?
}

Jeanne Demoulin

\section{To cite this version:}

Jeanne Demoulin. Stéphane Beaud et Gérard Mauger (dir.), Une génération sacrifiée ?. Lectures, 2016, 10.4000/lectures.23042 . hal-03086208

\section{HAL Id: hal-03086208 \\ https://hal.parisnanterre.fr/hal-03086208}

Submitted on 6 Jan 2021

HAL is a multi-disciplinary open access archive for the deposit and dissemination of scientific research documents, whether they are published or not. The documents may come from teaching and research institutions in France or abroad, or from public or private research centers.
L'archive ouverte pluridisciplinaire HAL, est destinée au dépôt et à la diffusion de documents scientifiques de niveau recherche, publiés ou non, émanant des établissements d'enseignement et de recherche français ou étrangers, des laboratoires publics ou privés. 


\title{
Stéphane Beaud et Gérard Mauger (dir.), Une génération sacrifiée?
}

\author{
Jeanne Demoulin
}

1 Cet ouvrage s'inscrit dans la lignée de travaux de recherche qui entendent analyser les recompositions à l'œuvre dans les classes populaires. Comme l'indiquent les auteurs en introduction, il fait partie de ces écrits qui « témoignent du renouvellement de l'intérêt des sociologues pour les classes populaires depuis une vingtaine d'années » (p. 7). Plus exactement, il vient alimenter les réflexions autour d'une catégorie spécifique, les jeunes, en centrant la focale sur les effets de la « crise profonde et durable du système scolaire, du marché du travail et de l'offre de biens symbolique » (p. 8).

2 Les onze contributions, regroupées en trois parties et encadrées par une introduction et une postface, rendent compte d'enquêtes empiriques de «jeunes sociologues » qui ont « pour la plupart » suivi les séminaires des deux directeurs de l'ouvrage à l'ENS ou à l'EHESS (p.15). Construites chacune à partir d'une thématique spécifique, elles constituent autant d'entrées qui permettent au lecteur d'appréhender la diversité des trajectoires des jeunes. S'il ne s'agit pas de se centrer uniquement sur les «jeunes des cités » mais de donner également à voir d'autres figures, l'ouvrage ne prétend pas à l'exhaustivité. En effet, en cohérence avec l'angle théorique choisi et exposé dans l'introduction, ce sont les effets d'une triple «crise» qui sont au cœur de l'investigation. C'est pourquoi l'ouvrage rend essentiellement compte de trajectoires de jeunes pris dans des processus de disqualification sociale.

3 La première partie, "Effets de génération. Désindustrialisation et massification scolaire », regroupe deux contributions qui analysent la manière dont les valeurs et les styles de vie portés par les jeunes hier et aujourd'hui constituent ou non des ressources pour leur intégration sociale. Elles explorent dans le même temps la nature et les caractéristiques du type d'intégration sociale en jeu ainsi que les ruptures qui s'opèrent en conséquence entre les générations dans les milieux populaires contemporains. Vincent Burckel montre ainsi comment deux générations d'hommes, isolées l'une de l'autre par l'effondrement de l'industrie minière et de la sidérurgie, se font face dans la «Lorraine du fer ». Par le prisme de la figure d'Hassan, un « vieux jeune » pris entre ces 
deux générations, il montre comment la virilité se trouve investie différemment par les vieux «hommes du fer " d'un côté et les «jeunes précaires» de l'autre. Si, pour ces deux groupes, les comportements virils ont un effet intégrateur, c'est «la résistance physique et morale au travail » (p. 30) qui est mise en avant par les premiers tandis que les deuxièmes se rapprochent davantage du «monde des bandes » et de la culture de la rue (p. 26): l'affirmation de valeurs de virilité vient alors compenser la "désaffiliation » à laquelle les jeunes sont confrontés. Dans le deuxième chapitre, Gérard Mauger centre son propos sur une comparaison entre les "styles de vie déviants » des jeunes des années 1970 et ceux d'aujourd'hui, en l'occurrence entre le "monde des bandes" et la "bohème populaire». Selon l'auteur, les ressources acquises par les jeunes adoptant des styles de vie déviants leur permettaient hier, entre autres, une intégration au marché du travail, tandis qu'elles viennent aujourd'hui compenser leur exclusion ou leur mise à l'écart du système scolaire et de l'emploi.

4 La deuxième partie, "Désarroi des jeunes hommes des classes populaires ", poursuit l'examen des effets de l'évolution de la reconnaissance sociale des valeurs de virilité. Elle interroge les conséquences d'une telle évolution sur l'intégration sociale des hommes à partir de trois enquêtes complémentaires. Benoît Coquard montre certains effets de la dévaluation des valeurs de virilité sur le marché du travail et sur le marché matrimonial auprès des jeunes sociabilisés au sein de milieux populaires dans lesquels ces valeurs continuent de structurer les rapports. Il met dans le même temps en évidence la manière dont les jeunes intériorisent les critères du succès et la manière dont ceux qui ne parviennent pas à s'y conformer « individualisent» (p. 54), en miroir, leur propre échec et rendent un jugement sans appel sur leur « vie de merde» (p. 61). Thomas Beaubreuil revient quant à lui sur ces "jeunes des halls" en décrivant comment ce style de vie se développe "dans l'interstice (dé)structurant ou (dé)socialisant produit par l'absence d'emplois et de revenus» (p. 81). Enfin, Alkim Oualhaci montre comment des jeunes déqualifiés peuvent retrouver une estime de soi par une pratique sportive, en l'occurrence celle de la boxe thaïlandaise, et plus spécifiquement par l'appartenance à un club qui promeut une stricte discipline des corps et des esprits. Une légère entorse à la thématique de cette partie de l'ouvrage est ici réalisée puisque l'auteur s'intéresse également aux jeunes filles qui, quoique peu nombreuses à s'investir pleinement dans la pratique étudiée, trouvent également au sein du club une manière de restaurer leur dignité et leur honneur.

La troisième partie, «Figures de jeunes femmes de classes populaires », est centrée sur les liens entre parcours scolaires et insertion professionnelle et entend ainsi prendre à rebours l'idée selon laquelle les jeunes femmes «s'en sortent » mieux que les jeunes hommes. À partir de l'exploitation secondaire de l'enquête "Génération 1998 " du Centre d'études et de recherches sur les qualifications, Ugo Palheta souhaite interroger à nouveaux frais ce "tableau avantageux, sinon idyllique d'une émancipation par l'école des femmes issues des classes populaires» (p.100). Il montre que poursuite d'études ne rime pas automatiquement avec réussite professionnelle, notamment parce que les filles sont nombreuses à poursuivre leurs études dans des filières qui offrent peu de débouchés professionnels ou des débouchés moins "rentables ». Sophie Orange s'intéresse pour sa part aux jeunes filles diplômées qui choisissent de rester dans leur milieu d'origine après l'obtention d'un diplôme de l'enseignement supérieur. Elle analyse ainsi les arrangements et adaptations opérées par ces jeunes "pour tenir ensemble l'impératif des études longues et la combinaison de l'emploi salarié féminin avec la maternité » (p.114). Selon l'auteure, l'«inertie» de ces jeunes filles ne peut 
s'accorder avec un parcours émancipatoire dans la mesure où elle témoigne de l'intériorisation et de la mise en œuvre de ces trois types de normes.

6 Dans la quatrième et dernière partie, "Engagements et désengagements politiques et syndicaux ", les chapitres apportent des éléments spécifiques sur les dynamiques contradictoires qui caractérisent le rapport des jeunes aux syndicats et à la politique. Dans le même temps, certaines entrent en écho avec nombre des contributions précédentes. Le chapitre de Charles Berthonneau et celui de Martin Thibault, qui étudie le rapport aux syndicats par le biais d'enquêtes à la CGT et à la RATP, complètent ainsi par exemple les analyses sur les "effets de génération", qui passent par un rapport spécifique au travail et à la virilité. Lorenzo Barrault-Stella et Clémentine Berjaud s'intéressent à une unique génération de lycéens et à leurs rapports au vote lors des élections présidentielles de 2012 ; ils montrent également les tensions et oppositions qui structurent les relations entre les membres d'une même classe sociale. Samuel Bouron et Pierig Humeau participent tout autant à cet effort de complexification en rendant compte de deux trajectoires d'engagement a priori fort éloignées l'une de l'autre, celles des punks et celles des identitaires.

$7 \mathrm{Au}$ terme de l'ouvrage, le lecteur est familiarisé avec un large éventail de cas, documentés dans le détail grâce à l'abondant matériel empirique mobilisé par chacun des auteurs. Il dispose ainsi d'éléments d'analyse et de compréhension relativement précis pour appréhender les jeunes des classes populaires. Dans le même temps, une impression d'uniformité et de répétition se dégage du livre. Si les contributions sont ancrées dans des terrains divers et étudient des jeunes aux profils variés, les analyses sont largement centrées sur des jeunes considérés comme "déviants», ou pris dans une spirale de déclassement ou de déqualification sociale. Sans ignorer ou remettre en question le fait que de tels phénomènes sont à l'œuvre dans les classes populaires, voire qu'ils structurent les modes de socialisation et d'intégration sociale de nombre de jeunes, on peut se demander si ces phénomènes "avalent» de manière aussi unilatérale les jeunes des classes populaires. Dit autrement, et pour reprendre les mots du titre, la génération toute entière s'est-elle trouvée "sacrifiée »? On sait que la réponse est négative. L'ouvrage aurait certainement gagné à présenter des cas contradictoires, afin de donner un panorama peut-être moins homogène.

Sur un plan plus méthodologique, j’ai déjà souligné la richesse des enquêtes empiriques rassemblées ici. L'ouvrage est essentiellement composé d'enquêtes ethnographiques (9 sur 11, comme cela est rappelé dans l'« avertissement » liminaire) et c'est ce qui fait en partie sa richesse: les différentes contributions racontent et donnent à voir concrètement, à grand renforts d'exemples, le quotidien des enquêtés et le discours sur soi qu'ils élaborent. Toutefois, les auteurs ne disent rien ou très peu de choses des conditions de leurs enquêtes ni des effets de leurs postures et méthodologies sur les résultats qu'ils avancent. Cela ne manque pas de soulever des interrogations sur la portée de ces résultats. Si l'on apprend par exemple que l'un des auteurs a emménagé dans le quartier étudié, qu'un autre participe aux entraînements de boxe parmi les jeunes qu'il étudie, ou que d'autres ont suivi pendant plusieurs mois des jeunes d'un lycée professionnel, ces positions ne donnent lieu à aucune analyse ou retour réflexif alors même que la nécessité d'un tel effort est soulignée dans l'introduction (note 36, p. 207). Aussi jeune soit-il ou soit-elle, le ou la sociologue qui a « réussi » appartient à un autre monde que celui des enquêtés. Dès lors, comment ces derniers se donnent-t-il à voir face à eux ? Plus largement, comment les rapports de classes, de genre et de race 
jouent-ils dans la relation d'enquête et conditionnent-ils le recueil et l'analyse des données? Alors que les auteurs insistent sur l'importance de la représentation dans la socialisation des jeunes, qu'en est-il de cette représentation dans leurs rapports aux enquêteurs? Si l'on ne doute pas que les auteurs aient mené ailleurs ce travail de réflexivité et d'objectivation, il aurait été sinon nécessaire du moins instructif d'en rendre compte dans cet ouvrage.

\section{AUTHOR}

\section{JEANNE DEMOULIN}

Sociologue et urbaniste, docteure de l'Université Paris-Ouest Nanterre-La Défense et attachée temporaire d'enseignement et d'urbanisme à l'École d'urbanisme de Paris / Lab'urba. 